\title{
An Approach to Spectrum Sensing in Cognitive Radio
}

\author{
Prabhat Sharma ${ }^{\# 1}$, Jai Karan Singh ${ }^{\# 2}$ \\ ${ }^{\#}$ Department of Electronics \& Communication
}

\begin{abstract}
Recent research shows that more than $70 \%$ of the available spectrum is not utilized efficiently. The bandwidth becomes expensive due to a shortage of frequencies. Therefore for efficient utilization of spectrum, we need to sniff the spectrum to determine whether it is being used by primary user or not. The term cognitive radio refers to the adoption of radio parameters using the sensed information of the spectrum. There are various spectrum sensing techniques proposed in the literature but still there is room for researchers in this field to explore more sophisticated approaches. There are three major categories of spectrum sensing techniques; transmitter detection, receiver detection and interference temperature detection. This thesis presents a survey of techniques suggested in the literature for spectrum sensing with a performance analysis of transmitter-based detection techniques.
\end{abstract}

Keywords-Include at least 5 keywords or phrases

\section{INTRODUCTION}

The radio frequency spectrum is a scarce natural resource and its efficient use is of the utmost importance. The spectrum bands are usually licensed to certain services, such as mobile, fixed, broadcast, and satellite, to avoid harmful interference between different networks to affect users. Most spectrum bands are allocated to certain services but worldwide spectrum occupancy measurements show that only portions of the spectrum band are fully used.

Moreover, there are large temporal and spatial variations in the spectrum occupancy. In the development of future wireless systems the spectrum utilization functionalities will play a key role due to the scarcity of unallocated spectrum. Moreover, the trend in wireless communication systems is going from fully centralized systems into the direction of self-organizing systems where individual nodes can instantaneously establish ad hoc networks whose structure is changing over time. Cognitive radios, with the capabilities to sense the operating environment, learn and adapt in real time according to environment creating a form of mesh network, are seen as a promising technology.

The cognitive radio presents a very lucrative area of the research field. Inefficient spectrum utilization is the driving force behind cognitive radio and adopting it can lead to a reduction of spectrum scarcity and better utilization of the spectrum resources. Spectrum Sensing i.e. checking the frequency spectrum for empty bands forms the foremost part of the cognitive radio. There are number of schemes for spectrum sensing like energy detector and matched filter. But the former functions properly for higher signal to noise ratio (SNR) value whereas the latter's complexity is very high. These constraints led to implementing a detector which performed well under low SNR conditions as well and with complexity not as high as the matched filter. Cyclo-stationary detector turned out to be the choice for such specifications [1].

In co-operative sensing (decision from number of users taken into consideration), number of users lead to more overhead and thus takes time for final decision. Hence better decision cost us time and efficiency. Lowering the detection threshold increases the detection as well as the chances of false detection. Thus one cannot lower the threshold value at will.

\section{LITERATURE SURVEY}

The main challenge to the Cognitive radios is the spectrum sensing. In spectrum sensing there is a need to find spectrum holes in the radio environment for CR users. However it is difficult for CR to have a direct measurement of channel between primary transmitter and receiver [2]. A CR can not transmit and detect the radio environment simultaneously, thus, we need such spectrum sensing techniques that take less time for sensing the radio environment. In literature the spectrum sensing techniques have been classified in the following three categories [2]. 


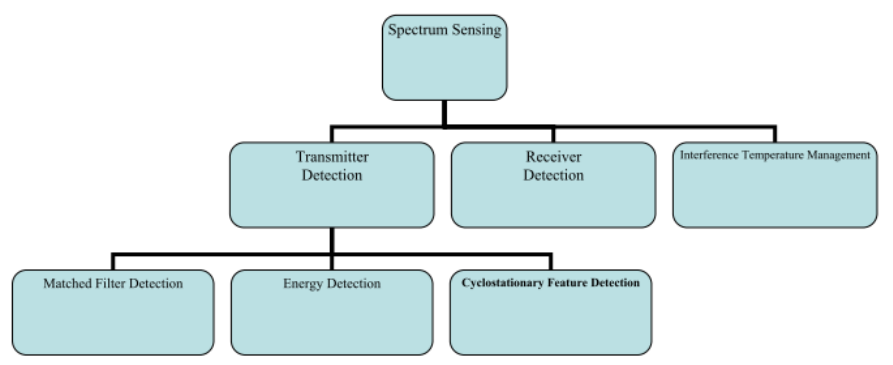

Fig 1. Spectrum Sensing Technique

In transmitter detection we have to find the primary transmitters that are transmitting at any given time. Hypothesis model for transmitter detection is defined in [3] that is, the signal received (detected) by the $\mathrm{CR}$ (secondary) user is

$$
\begin{aligned}
& x(t)=\left\{n(t) H_{0}\right. \\
& x(t)=\left\{h s(t)+n(t) H_{1}\right.
\end{aligned}
$$

Where $\mathrm{x}(\mathrm{t})$ is the signal received by $\mathrm{CR}, \mathrm{s}(\mathrm{t})$ is the transmitted signal of primary user, $\mathrm{n}(\mathrm{t})$ is additive white Gaussian noise, and $\mathrm{h}$ is the amplitude gain of the channel.

\subsection{Matched Filter Detection:}

A matched filter is a linear filter designed to provide the maximum signal-to noise ratio at its output for a given transmitted waveform [4]. Figure 2.1 depicts the block diagram of matched filter. The signal received by CR is input to matched filter which is $r(t)=s(t)+n(t)$. The matched filter convolves the $r(t)$ with $h(t)$ where $h$ $(t)=s(T-t+\tau)$. Finally the output of matched filter is compared with a threshold $\lambda$ to decide whether the primary user is present or not.

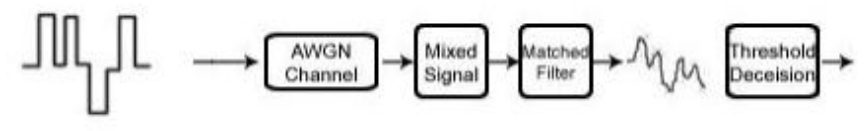

Figure 2.1 Block Diagram of Matched Filter

A Matched filter is an optimal detector in an AWGN channel if the waveform of primary user is previously known by CR. It means that CR should have knowledge about the waveform of primary user such as modulation type and order, the pulse shape and the packet format. So if CR doesn't have this type of prior information then it's difficult to detect the primary user. We can still use Matched Filter Detection because in most of the communication networks we can achieve this coherency by introducing pilots, preambles, synchronization word, or spreading codes in the waveform of primary users. Still there are limitations in matched filter because each CR should have the information of all the primary users present in the radio environment. Advantage of matched filter is that it takes less time for high processing gain. However major drawback of Matched Filter is at a CR would need a dedicated receiver for every primary user class [5].

\subsection{Energy Detection}

If CR can't have sufficient information about primary user's waveform, then the matched filter is not the optimal choice. However if it is aware of the power of the random Gaussian noise, then energy detector is optimal [2]. In [7] the authors proposed the energy detector as shown in Figure 2.2. The input band pass filter selects the center frequency fs and bandwidth of interest $\mathrm{W}$. The filter is followed by a squaring device to measure the received energy then the integrator determines the observation interval, T. Finally the output of the integrator, $\mathrm{Y}$ is compared with a threshold, $\lambda$ to decide whether primary user is present or not. 


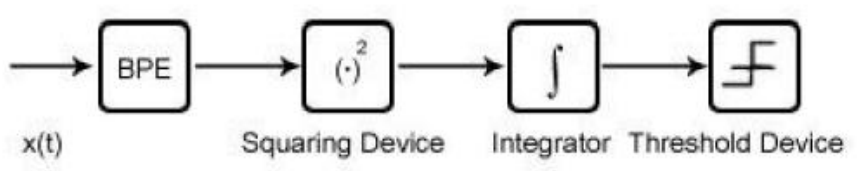

Figure 2.2 Block Diagram of Energy Detector

One of the main problems of energy detection is that performance is susceptible to uncertainty in noise power. It cannot differentiate between signal power and noise power rather it just tells us about absence or presence of the primary user.

\subsection{Cyclostationary Feature Detection}

Modulated signals are in general coupled with sine wave carriers, pulse trains, repeating spreading, hopping sequences, or cyclic prefixes, which result in built-in periodicity [4]. Even though the data is stationary random process, these modulated signals are characterized as Cyclostationary, since their statistics, mean and autocorrelation, exhibits periodicity. These features are detected by analyzing a spectral correlation function. The periodicity is provided for signal format so that receiver can use it for parameter estimation like pulse timing, carrier phase etc. This periodicity can be used in the detection of random signals with a particular type of modulation with the noise and other modulated signals.

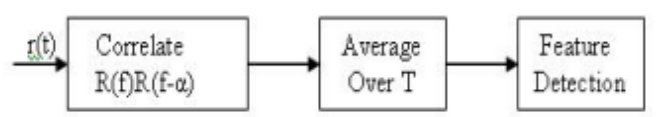

Figure 2.3 Block Diagram of Cyclostationary Feature Detector

\section{Cooperative Vs Non Cooperative}

The detection behavior can be categorized into two main branches, Non cooperative and cooperative. In non cooperative detection behavior cognitive radio user can detect the signal of primary transmitter by its own observation and analysis independent of the other cognitive radio users. While in Cooperative detection behavior the information from many cognitive radio users are combined to detect the primary user.

Moreover, Cooperative behavior helps to overcome the multi path fading and shadowing effect that will increase its usability. There are two ways for the implementation of cooperative detection, centralized and distributed. In Centralized Cooperative detection mechanism the base station is responsible for gathering all information from other cognitive radio users to detect the primary users.

\section{METHODOLOGY USED}

First of all we need primary user waveform on which we can apply different spectrum sensing techniques. Transmitter can have different transmitting parameters like they can have different operating frequency, different modulation scheme.

\subsection{Primary Transmitter}

Step 1: The system parameters are set in this step. The parameters are: (i) the operating frequency, 'freq'; (ii) the sampling frequency, 'Fs'; (iii) number of samples per symbol period, 'L'; (iv) the sampling period, 'Ts'; (v) roll-off factor for the (square-root) raised cosine filters, 'alpha'; (vi) N+1 is the length of the square-root raised cosine filter, 'N'; (vii) signal to noise ratio, 'snr'; (viii) channel impulse response, 'h'.

Step 2: This is any piece of information (a text file, a sampled speech signal, a coded image, ......) that is converted to sequence of bits. Here are two options either take input from the user to transmit or use default data sequence.

Step 3: This a square-root raised-cosine filters with roll-off factor $\alpha$. Here, $\alpha$ is set equal to 0.5 . In the real world, the transmit signal is continuous time. Since in computer simulation, we can only have sampled signals, we approximate continuous-time signals by a dense grid of samples. Here, we have $\mathrm{L}=100$ samples per symbol period. The function ' $s r$ cos $p$ ' generates a square-root raised-cosine pulse, for the transmit filter, $\mathrm{pT}(\mathrm{t})$. The output of this step is $\overline{\mathrm{Y}}$. 
Step 4: Modulation is done to generate an RF (radio frequency) signal for transmission through channel. Here two modulation techniques BPSK (Binary Phase Shift Keying) and QPSK (Qaurdrature Phase Shift Keying) are available. It depends on type of primary transmitter that whether to use BPSK or QPSK.

Step 5: This is characterized by an impulse response $c(t)$ and an additive noise. Here, we have chosen $c(t)=$ $\delta(t)$ which in the discrete domain becomes $c=1$. If the channel is multipath, e.g., with the impulse response $\mathrm{c}(\mathrm{t})=\mathrm{a} 0 \delta(\mathrm{t}-\mathrm{t} 0)+\mathrm{a} 1 \delta(\mathrm{t}-\mathrm{t} 1)$, it has the equivalent discrete domain $\mathrm{c}=[\mathrm{zeros}(\mathrm{N} 0,1) ; \mathrm{a} 0$; zeros(N1,1); a1], where N0 and $\mathrm{N} 1$ are $\mathrm{t} 0$ and $\mathrm{t} 1$ in unit of Ts.

Step 6: The channel noise is assumed to be Additive White Gaussian with signal strength 2dB. In MATLAB 'awgn' function is used for this purpose.

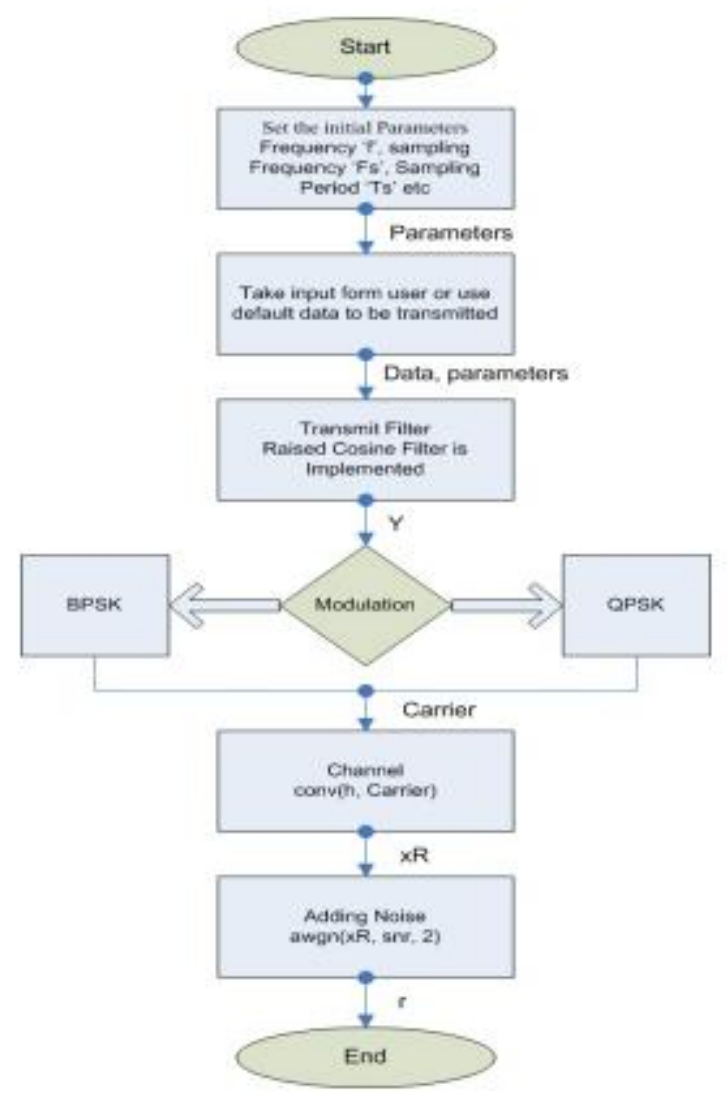

Fig 3.1 Flow chart for Implementation of Primary Transmitter

\subsection{Energy Detection}

Step 1: First estimate Power Spectral Density (PSD) by using periodogram function in MATLAB.

Pxx $=$ Periodogram $(r)$

Step 2: The power spectral density (PSD) is intended for continuous spectra. The integral of the PSD over a given frequency band computes the average power in the signal over that frequency band.

Hpsd=Dspdata.psd(Pxx)

Step 3: Now one frequency component takes almost 20 points in MATLAB. So for each frequency there points are summed and get the result.

Step 4: On experimental basis when results at low and high SNR are compared then threshold $\lambda$ is set to be 5000 .

Step 5: Finally the output of the integrator, $\mathrm{Y}$ is compared with a threshold value $\lambda$ to decide whether primary user is present or not. 


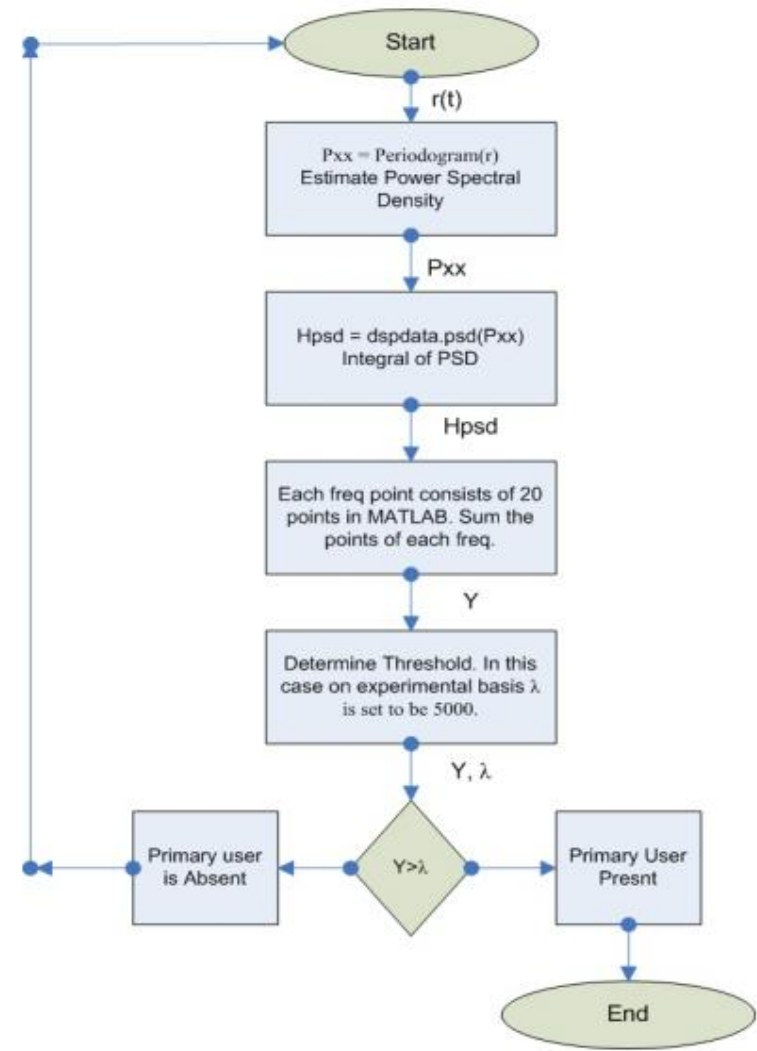

Fig 3.2 Flow chart for Implementation of Energy Detector

\section{IMPLEMENTATION AND RESULT}

Figure 4.1 shows the output of energy detector when there is a primary user at $200 \mathrm{~Hz}$ using BPSK is present with very good SNR. It's very clear in the figure that there is peak at exactly $200 \mathrm{~Hz}$. So energy detector compared this peak with threshold value, in this case its greater then threshold. Hence, energy detector said that primary user is present at $200 \mathrm{~Hz}$.

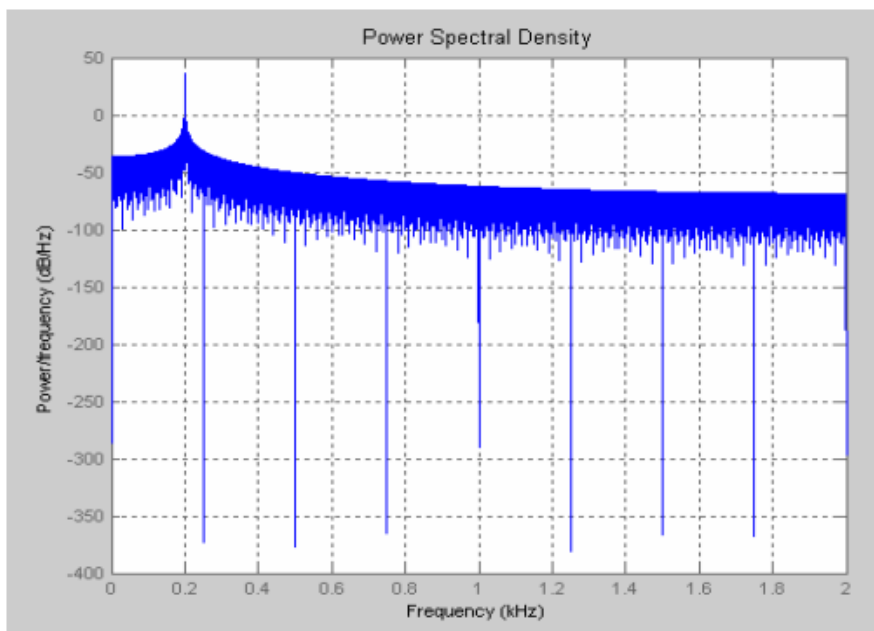

Fig 4.1 Energy Detector Output at SNR 30dB for BPSK when primary user is present at $200 \mathrm{~Hz}$

Figure 4.2 shows the correlation of received signal with signal generated at cognitive radio under good SNR conditions 


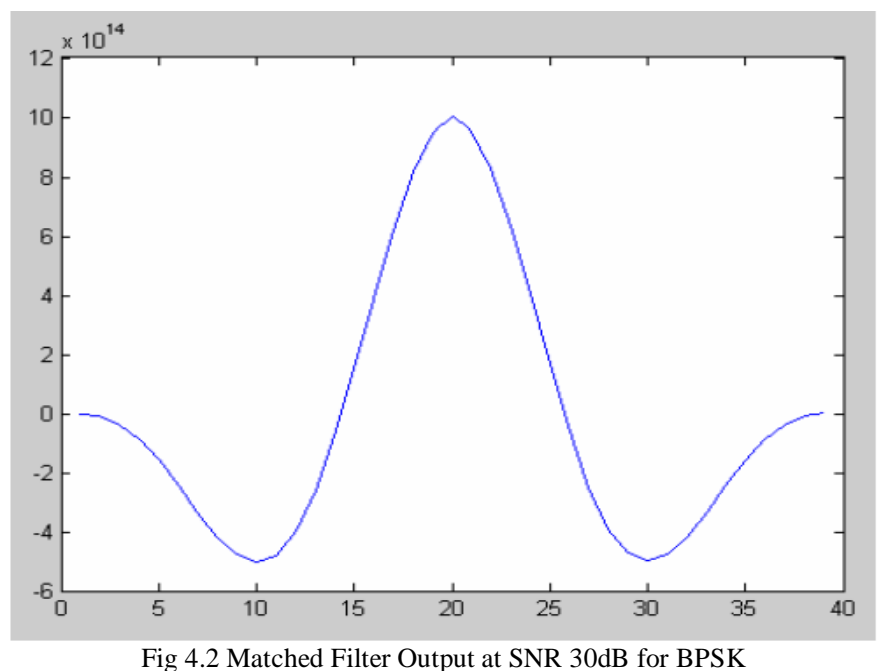

Figure 4.3 shows the output of cyclostationary feature detection when there is a primary user at $200 \mathrm{~Hz}$ using BPSK is present with very good SNR. It's very clear in the figure that there is peak in the center and there is a peak at double of the frequency as well. Now we have to compare second peak with threshold value. So cyclostationary feature detection compared value of each peak with threshold, in this case its greater then threshold at 400 . Hence, cyclostationary feature detection said that primary user is present at $200 \mathrm{~Hz}$.

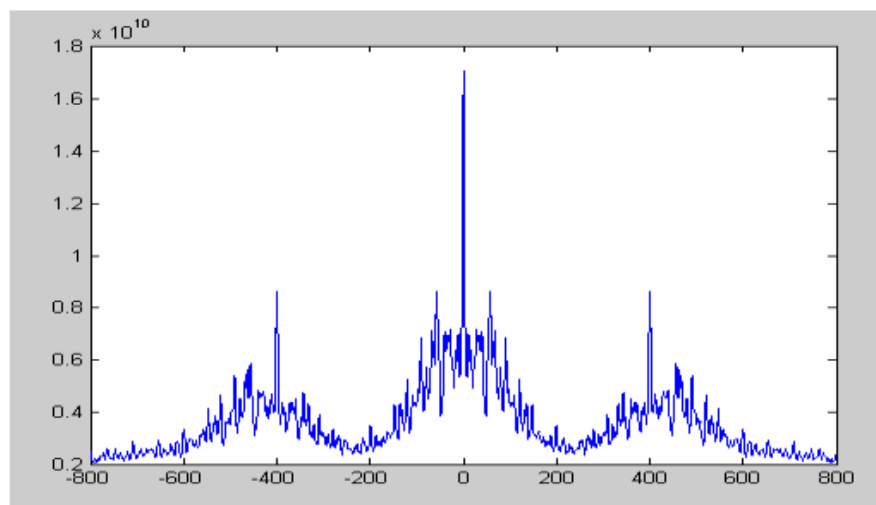

Fig 4.3 Cyclostationary Feature Detector Output at SNR 30dB for BPSK when primary user is present at $200 \mathrm{~Hz}$

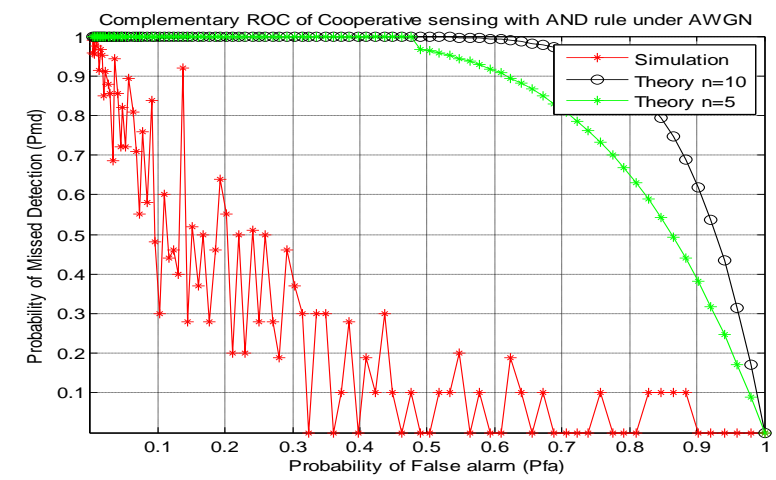

Fig 4.4 Complementry ROC of Cooperative sensing with rule under AWGN

\section{CONCLUSION AND FUTURE SCOPE}

As the demand of radio spectrum increases in past few years and licensed bands are used inefficiently, improvement in the existing spectrum access policy is expected. Dynamic spectrum access is imagine to resolve the spectrum shortage by allowing unlicensed users to dynamically utilize spectrum holes across the licensed spectrum on noninterfering basis.

This research was aimed towards the detection and classification of primary user's waveform in cognitive radio networks. The primary requirement of a spectrum sensing system is its real time processing 
and decision making. The proposed methodology has been implemented on a desktop PC and requires MATLAB support for simulation. Its implementation can be done on FPGA kit or DSP processor.

\section{References}

[1] ET Docket No. 03-222 Notice of proposed rule making and order, December 2003

[2] I.F Akyildiz,W Lee, M.C Vuran, S Mohanty,"Next Generation/ Dynamic spectrum access/cognitive radio wireless networks: A survey" Computer Networks 50(2006) 2127-2159, May 2006

[3] A. Ghasemi and E. S. Sousa,"Collaborative Spectrum Sensing for Opportunistic Access in Fading Environment", in Proc. IEEE DySPAN, pp. 131-136, Nov. 2005

[4] Amir Ghasemi, Elvino S. Sousa: "Spectrum Sensing in Cognitive Radio Networks: Requirements, Challenges and Design Trade-offs", IEEE Communication Magzine April 2008. page(s): 32-39.

[5] Tandra, R. Sahai, A.: SNR Walls for Feature Detectors, New Frontiers in Dynamic Spectrum Access Networks, April 2007. page(s): 559-570. 